\title{
Holographic modified gravity
}

\author{
M.R. Setare * \\ Department of Science, Payame Noor University. Bijar, Iran
}

\begin{abstract}
In this paper we study cosmological application of holographic dark energy density in the modified gravity framework. We employ the holographic model of dark energy to obtain the equation of state for the holographic energy density in spatially flat universe. Our calculation show, taking $\Omega_{\Lambda}=0.73$ for the present time, it is possible to have $w_{\Lambda}$ crossing -1 . This implies that one can generate phantom-like equation of state from a holographic dark energy model in flat universe in the modified gravity cosmology framework. Also we develop a reconstruction scheme for the modified gravity with $f(R)$ action.
\end{abstract}

\footnotetext{
*E-mail: rezakord@ipm.ir
} 


\section{Introduction}

Nowadays it is strongly believed that the universe is experiencing an accelerated expansion. Recent observations from type Ia supernovae [1] in associated with Large Scale Structure [2] and Cosmic Microwave Background anisotropies [3] have provided main evidence for this cosmic acceleration. In order to explain why the cosmic acceleration happens, many theories have been proposed. It is the most accepted idea that a mysterious dominant component, dark energy, with negative pressure, leads to this cosmic acceleration, though its nature and cosmological origin still remain enigmatic at present. An alternative proposal for dark energy is the dynamical dark energy scenario. The cosmological constant puzzles may be better interpreted by assuming that the vacuum energy is canceled to exactly zero by some unknown mechanism and introducing a dark energy component with a dynamically variable equation of state. The dynamical dark energy proposal is often realized by some scalar field mechanism which suggests that the energy form with negative pressure is provided by a scalar field evolving down a proper potential. In recent years, many string theorists have devoted to understand and shed light on the cosmological constant or dark energy within the string framework. The famous KachruKallosh-Linde-Trivedi (KKLT) model [4] is a typical example, which tries to construct metastable de Sitter vacua in the light of type IIB string theory. Furthermore, string landscape idea [5] has been proposed for shedding light on the cosmological constant problem based upon the anthropic principle and multiverse speculation. Although we are lacking a quantum gravity theory today, we still can make some attempts to probe the nature of dark energy according to some principles of quantum gravity. The holographic dark energy model is just an appropriate example, which is constructed in the light of the holographic principle of quantum gravity theory. That is to say, the holographic dark energy model possesses some significant features of an underlying theory of dark energy. Currently, an interesting attempt for probing the nature of dark energy within the framework of quantum gravity is the so-called "holographic dark energy" proposal $[6,7,8,9]$. It is well known that the holographic principle is an important result of the recent researches for exploring the quantum gravity (or string theory) [10]. This principle is enlightened by investigations of the quantum property of black holes. Roughly speaking, in a quantum gravity system, the conventional local quantum field theory will break down. The reason is rather simple: For a quantum gravity system, the conventional local quantum field theory contains too many degrees of freedom, and such many degrees of freedom will lead to the formation of black hole so as to break the effectiveness of the quantum field theory.

For an effective field theory in a box of size $L$, with UV cut-off $\Lambda$ the entropy $S$ scales extensively, $S \sim L^{3} \Lambda^{3}$. However, the peculiar thermodynamics of black hole [11] has led Bekenstein to postulate that the maximum entropy in a box of volume $L^{3}$ behaves nonextensively, growing only as the area of the box, i.e. there is a so-called Bekenstein entropy bound, $S \leq S_{B H} \equiv \pi M_{P}^{2} L^{2}$. This nonextensive scaling suggests that quantum field theory breaks down in large volume. To reconcile this breakdown with the success of local quantum field theory in describing observed particle phenomenology, Cohen et al. [6] proposed a more restrictive bound - the energy bound. They pointed out that in quantum field theory a short distance (UV) cut-off is related to a long distance (IR) cut-off due to the limit set by forming a black hole. In other words, if the quantum zero-point energy density $\rho_{\Lambda}$ is relevant to a UV cut-off $\Lambda$, the total energy of the whole system with size $L$ should not exceed the mass of a black hole of the same size, thus we 
have $L^{3} \rho_{\Lambda} \leq L M_{P}^{2}$. This means that the maximum entropy is in order of $S_{B H}^{3 / 4}$. When we take the whole universe into account, the vacuum energy related to this holographic principle [10] is viewed as dark energy, usually dubbed holographic dark energy. Such a holographic dark energy looks reasonable, since it may provide simultaneously natural solutions to both dark energy problems as demonstrated in Ref.[9]. The holographic dark energy model has been tested and constrained by various astronomical observations [12]. Furthermore, the holographic dark energy model has been extended to include the spatial curvature contribution, i.e. the holographic dark energy model in non-flat space [13]. Because the holographic energy density belongs to a dynamical cosmological constant, we need a dynamical frame to accommodate it instead of general relativity. Therefore it is worthwhile to investigate the holographic energy density in the framework of the Brans-Dicke theory $[14,15,16,17]$. Einstein's theory of gravity may not describe gravity at very high energy. The simplest alternative to general relativity is Brans-Dicke scalartensor theory [18]. Modified gravity provides the natural gravitational alternative for dark energy [19]. Moreover, modified gravity present natural unification of the earlytime inflation and late-time acceleration thanks to different role of gravitational terms relevant at small and at large curvature. Also modified gravity may naturally describe the transition from non-phantom phase to phantom one without necessity to introduce the exotic matter. But among the most popular modified gravities which may successfully describe the cosmic speed-up is $F(R)$ gravity. Very simple versions of such theory like $1 / R[20]$ and $1 / R+R^{2}[21]$ may lead to the effective quintessence/phantom late-time universe (to see solar system constraints on modified dark energy models refer to [23]). Another theory proposed as gravitational dark energy is scalar-Gauss-Bonnet gravity [22] which is closely related with low-energy string effective action.

In present paper, using the holographic model of dark energy in spatially flat universe, we obtain equation of state for holographic dark energy density in framework of modified gravity for a universe enveloped by $R_{h}$ as the system's IR cut-off. The current available observational data imply that the holographic vacuum energy behaves as phantom-type dark energy, i.e. the equation-of-state of dark energy crosses the cosmological-constant boundary $w=-1$ during the evolution history. We show this phantomic description of the holographic dark energy in flat universe with $0.21 \leq c \leq 2.1$. Also we develop a reconstruction scheme for the modified gravity with $f(R)$ action, the known holographic energy density is used for this reconstruction.

\section{Modified gravity and holographic dark energy}

The action of modified gravity is given by

$$
S=\int d^{4} x\left[f(R)+L_{m}\right]
$$

where $L_{m}$ is the matter Lagrangian density. The equivalent form of above action is [19]

$$
S=\int d^{4} x \sqrt{-g}\left[P(\phi) R+Q(\phi)+L_{m}\right]
$$

where $P$ and $Q$ are proper functions of the scalar field $\phi$. By the variation of the action (2) with respect to the $\phi$, we obtain

$$
P^{\prime}(\phi) R+Q^{\prime}(\phi)=0
$$


which may be solved with respect to $\phi$ :

$$
\phi=\phi(R)
$$

By the variation of the action (2) with respect to the metric $g_{\mu \nu}$, one can obtain

$$
\frac{-1}{2} g_{\mu \nu}[P(\phi) R+Q(\phi)]-R_{\mu \nu} P(\phi)+\nabla_{\mu} \nabla_{\nu} P(\phi)-g_{\mu \nu} \nabla^{2} P(\phi)+\frac{1}{2} T_{\mu \nu}=0
$$

where $T_{\mu \nu}$ is the energy-momentum tensor. The equations corresponding to standard spatially-flat FRW universe are

$$
\begin{gathered}
\rho=6 H^{2} P(\phi)+Q(\phi)+6 H \frac{d P(\phi)}{d t} \\
p=-\left(4 \dot{H}+6 H^{2}\right) P(\phi)-Q(\phi)-2 \frac{d^{2} P(\phi)}{d t^{2}}-4 H \frac{d P(\phi)}{d t}
\end{gathered}
$$

where, $p$ and $\rho$ are the pressure and energy density due to the scalar field in the modified gravity framework. By combining (6) and (7) and deleting $Q(\phi)$, we find

$$
p+\rho=-2 \frac{d^{2} P(\phi)}{d t^{2}}+2 H \frac{d P(\phi)}{d t}-4 \dot{H} P(\phi)
$$

Now we suggest a correspondence between the holographic dark energy scenario and the above modified dark energy model. The holographic energy density $\rho_{\Lambda}$ is chosen to be

$$
\rho_{\Lambda}=\frac{3 c^{2}}{R_{h}^{2}}
$$

where $c$ is a constant, and $R_{h}$ is the future event horizon given by

$$
R_{h}=a \int_{t}^{\infty} \frac{d t}{a}=a \int_{a}^{\infty} \frac{d a}{H a^{2}}
$$

The critical energy density, $\rho_{c r}$, is given by following relation

$$
\rho_{c r}=3 H^{2}
$$

Now we define the dimensionless dark energy as

$$
\Omega_{\Lambda}=\frac{\rho_{\Lambda}}{\rho_{c r}}=\frac{c^{2}}{R_{h}^{2} H^{2}}
$$

Using definition $\Omega_{\Lambda}$ and relation $(11), \dot{R}_{h}$ gets:

$$
\dot{R}_{h}=R_{h} H-1=\frac{c}{\sqrt{\Omega_{\Lambda}}}-1
$$

Let us consider the dark energy dominated universe. In this case the dark energy evolves according to its conservation law

$$
\dot{\rho}_{\Lambda}+3 H\left(\rho_{\Lambda}+P_{\Lambda}\right)=0
$$


By considering the definition of holographic energy density $\rho_{\Lambda}$, and using Eq.(13)one can find:

$$
\dot{\rho_{\Lambda}}=\frac{-2}{R_{h}}\left(\frac{c}{\sqrt{\Omega_{\Lambda}}}-1\right) \rho_{\Lambda}
$$

Substitute this relation into Eq.(14) we obtain

$$
w_{\Lambda}=-\left(\frac{1}{3}+\frac{2 \sqrt{\Omega_{\Lambda}}}{3 c}\right)
$$

A direct fit of the present available SNe Ia data with this holographic model indicates that the best fit result is $c=0.21$ [25]. Recently, by calculating the average equation of state of the dark energy and the angular scale of the acoustic oscillation from the BOOMERANG and WMAP data on the CMB to constrain the holographic dark energy model, the authors show that the reasonable result is $c \sim 0.7$ [26]. In the other hand, in the study of the constraints on the dark energy from the holographic connection to the small $l$ CMB suppression, an opposite result is derived, i.e. it implies the best fit result is $c=2.1$ [27]. Thus according to these studies $0.21 \leq c \leq 2.1$. Taking $\Omega_{\Lambda}=0.73$ for the present time, in the case of $c=0.21$, we obtain $w_{\Lambda}=-3.04$, in the other hand for $c=2.1$, one can obtain, $w_{\Lambda}=-0.6$. Using Eq.(16), one can see that by considering $c \leq \sqrt{\Omega_{\Lambda}}$ we obtain $w_{\Lambda} \leq-1$. Therefore taking $\Omega_{\Lambda}=0.73$ for the present time, it is possible to have $w_{\Lambda}$ crossing -1 .

As one can redefine the scalar field $\phi$ properly, we may choose

$$
\phi=t
$$

Now using Eqs.(9), (16), one can rewrite Eq.(8) as

$$
2 \frac{d^{2} P(t)}{d t^{2}}-2 H \frac{d P(t)}{d t}+4 \dot{H} P(t)+2 \Omega_{\Lambda} H^{2}\left(1-\frac{\sqrt{\Omega_{\Lambda}}}{c}\right)=0
$$

In principle, by solving Eq.(18) we find the form of $P(\phi)$. Using Eqs. (6), (9), we also find the form of $Q(\phi)$ as

$$
Q(\phi)=3 \Omega_{\Lambda} H^{2}-6 H^{2} P(\phi)-6 H \frac{d P(\phi)}{d t}
$$

\section{Modified gravity and its reconstruction from the holographic dark energy}

In this section we consider another approach [24] to realistic cosmology in holographic modified gravity. We start with general $f(R)$-gravity action (1) but without the matter term. For the spatially flat FRW universe we have

$$
\begin{gathered}
\rho=f(R)-6\left(\dot{H}+H^{2}-H \frac{d}{d t}\right) f^{\prime}(R) \\
p=f(R)-2\left(-\dot{H}-3 H^{2}+\frac{d^{2}}{d t^{2}}+2 H \frac{d}{d t}\right) f^{\prime}(R)
\end{gathered}
$$

where

$$
R=6 \dot{H}+12 H^{2}
$$


Again we use the holographic dark energy density and substitute Eq.(9) into Eq.(20)

$$
3 \Omega_{\Lambda} H^{2}=f(R)-6\left(\dot{H}+H^{2}-H \frac{d}{d t}\right) f^{\prime}(R)
$$

thus

$$
f(R)=3 \Omega_{\Lambda} H^{2}+6\left(\dot{H}+H^{2}-H \frac{d}{d t}\right) f^{\prime}(R)
$$

Using Eqs.(9), (16), and substituting $f(R)$ into Eq.(21) one can obtain

$$
2 \frac{d^{2}}{d t^{2}} f^{\prime}(R)-2 H \frac{d}{d t} f^{\prime}(R)+4 \dot{H} f^{\prime}(R)+2 \Omega_{\Lambda} H^{2}\left(1-\frac{\sqrt{\Omega_{\Lambda}}}{c}\right)=0
$$

or in another form

$$
2\left(f^{\prime \prime \prime} \dot{R}^{2}+f^{\prime \prime} \ddot{R}\right)-2 H f^{\prime \prime} \dot{R}+4 \dot{H} f^{\prime}+2 \Omega_{\Lambda} H^{2}\left(1-\frac{\sqrt{\Omega_{\Lambda}}}{c}\right)=0
$$

We shall consider the following simple solution

$$
a=a_{0}\left(t_{s}-t\right)^{h_{0}},
$$

where $a_{0}, h_{0}$ and $t_{s}$ are constant. Substituting Eq.(27) into Eq.(22), give us following relation for scalar curvature

$$
R=\frac{12 h_{0}^{2}-6 h_{0}}{\left(t_{s}-t\right)^{2}}
$$

Using Eqs.(10, 27) we can write

$$
R_{h}=a_{0}\left(t_{s}-t\right)^{h_{0}} \int_{t}^{t_{s}} \frac{d t}{a_{0}\left(t_{s}-t\right)^{h_{0}}}=\frac{t_{s}-t}{1-h_{0}}
$$

Now using definition $\rho_{\Lambda}$ and above relation we obtain the time behaviour of holographic dark energy as

$$
\rho_{\Lambda}=\frac{3 c^{2}}{R_{h}^{2}}=\frac{3 c^{2}\left(1-h_{0}\right)^{2}}{\left(t_{s}-t\right)^{2}}
$$

Substituting the above $\rho_{\Lambda}$ into Eq.(20), and using Eqs. $(27,28)$ one can obtain

$$
\frac{72 h_{0}^{2}\left(1-2 h_{0}\right)}{\left(t_{s}-t\right)^{4}} f^{\prime \prime}(R)-\frac{6 h_{0}\left(h_{0}-1\right)}{\left(t_{s}-t\right)^{2}} f^{\prime}(R)+f(R)=\frac{3 c^{2}\left(1-h_{0}\right)^{2}}{\left(t_{s}-t\right)^{2}}
$$

Again we use Eq.(28) and rewrite the above differential equation as following

$$
f^{\prime \prime}(R)+\frac{a}{R} f^{\prime}(R)+\frac{b}{R^{2}} f(R)=\frac{d}{R}
$$

where

$$
a=\frac{h_{0}-1}{2}, \quad b=\frac{1-2 h_{0}}{2}, \quad d=\frac{c^{2}\left(1-h_{0}\right)^{2}}{4 h_{0}}
$$

The solution of differential equation (32) is given by

$$
f(R)=C_{1} R^{\frac{1}{2}\left(\frac{3-h_{0}}{2}-\sqrt{\frac{\left(h_{0}-3\right)^{2}}{4}+4 h_{0}-2}\right)}+C_{2} R^{\frac{1}{2}\left(\frac{3-h_{0}}{2}+\sqrt{\frac{\left(h_{0}-3\right)^{2}}{4}+4 h_{0}-2}\right)}+\frac{c^{2}\left(1-h_{0}\right)^{2} R}{2 h_{0}^{2}}
$$


where $C_{1}, C_{2}$ are constant. Therefore, a consistent modified gravity whit holographic dark energy in flat space has the above form. Let us recall the two sufficient conditions which often lead to realistic models [23, 28]

$$
\lim _{R \rightarrow 0} f(R)=0
$$

this condition ensures the disappearance of the cosmological constant in the limit of flat space-time. One can see that (34) simply satisfy the above condition. In order that the accelerating expansion in the present universe could be generated, let us consider that $f(R)$ could be a small constant at present universe, that is,

$$
f\left(R_{0}\right)=-2 R_{0}, \quad f^{\prime}\left(R_{0}\right) \sim 0,
$$

where $R_{0} \sim\left(10^{-33} \mathrm{eV}\right)^{2}$ is current curvature [23]. By impose the conditions (36) on the solution (34) we can obtain the constants $C_{1}$ and $C_{2}$ as following

$$
\begin{aligned}
C_{1} & =\frac{-R_{0}^{1-u}}{u+v}\left[2 v+(v+1) \frac{c^{2}\left(1-h_{0}\right)^{2}}{2 h_{0}^{2}}\right] \\
C_{2} & =\frac{R_{0}^{1-v}}{u+v}\left[2 u-(1-u) \frac{c^{2}\left(1-h_{0}\right)^{2}}{2 h_{0}^{2}}\right]
\end{aligned}
$$

where

$$
u=\frac{1}{2}\left(\frac{3-h_{0}}{2}-\sqrt{\frac{\left(h_{0}-3\right)^{2}}{4}+4 h_{0}-2}\right), \quad v=\frac{1}{2}\left(\frac{3-h_{0}}{2}+\sqrt{\frac{\left(h_{0}-3\right)^{2}}{4}+4 h_{0}-2}\right)
$$

The model Eq.(34) is similar to the model of Eq.(12) in [29], similarly our model also leads to acceptable cosmic speed-up and is consistent with solar system tests.

\section{Conclusions}

Within the different candidates to play the role of the dark energy, the modified gravity, has emerged as a possible unification of dark matter and dark energy. In the present paper we have studied cosmological application of holographic dark energy density in the modified gravity framework. By considering the holographic energy density as a dynamical cosmological constant, we have obtained the equation of state for the holographic energy density in the modified gravity framework. We have shown if $c \leq \sqrt{\Omega_{\Lambda}}$, the holographic dark energy model also will behave like a phantom model of dark energy the amazing feature of which is that the equation of state of dark energy component $w_{\Lambda}$ crosses -1 . Hence, we see, the determining of the value of $c$ is a key point to the feature of the holographic dark energy and the ultimate fate of the universe as well. Finally we have developed a reconstruction scheme for modified gravity with $f(R)$ action. We have considered the energy density in Eq.(20) in holographic form, then by assumption a simple solution as Eq.(27) we could obtain a differential equation for $f(R)$, the solution of this differential equation give us a modified gravity action which is consistent with holographic dark energy scenario. 


\section{Acknowledgment}

The author would like to thank the referee because of his useful comments, which assisted to prepare better frame for this study.

\section{References}

[1] A. G. Riess et al. [Supernova Search Team Collaboration], Astron. J. 116, 1009 (1998) [astro-ph/9805201];

S. Perlmutter et al. [Supernova Cosmology Project Collaboration], Astrophys. J. 517, 565 (1999) [astro-ph/9812133];

P. Astier et al., Astron. Astrophys. 447, 31 (2006) [astro-ph/0510447].

[2] M. Tegmark et al. [SDSS Collaboration], Phys. Rev. D 69, 103501 (2004) [astroph/0310723];

K. Abazajian et al. [SDSS Collaboration], Astron. J. 128, 502 (2004) [astro$\mathrm{ph} / 0403325]$;

K. Abazajian et al. [SDSS Collaboration], Astron. J. 129, 1755 (2005) [astroph/0410239].

[3] D. N. Spergel et al. [WMAP Collaboration], Astrophys. J. Suppl. 148, 175 (2003) [astro-ph/0302209];

D. N. Spergel et al., astro-ph/0603449.

[4] S. Kachru, R. Kallosh, A. Linde and S. P. Trivedi, Phys. Rev. D 68, 046005 (2003) [hep-th/0301240].

[5] L. Susskind, hep-th/0302219.

[6] A. G. Cohen, D. B. Kaplan and A. E. Nelson, Phys. Rev. Lett. 82, 4971 (1999) [hep-th/9803132].

[7] P. Horava and D. Minic, Phys. Rev. Lett. 85, 1610 (2000) [hep-th/0001145];

S. D. Thomas, Phys. Rev. Lett. 89, 081301 (2002).

[8] S. D. H. Hsu, Phys. Lett. B 594, 13 (2004) [hep-th/0403052].

[9] M. Li, Phys. Lett. B 603, 1 (2004) [hep-th/0403127].

[10] G. 't Hooft, gr-qc/9310026;

L. Susskind, J. Math. Phys. 36, 6377 (1995) [hep-th/9409089].

[11] J. D. Bekenstein, Phys. Rev. D 7 (1973) 2333;

J. D. Bekenstein, Phys. Rev. D 49 (1994) 1912;

S. W. Hawking, Commun. Math. Phys. 43 (1975) 199;

S. W. Hawking, Phys. Rev. D 13 (1976) 191.

[12] Q. G. Huang and Y. G. Gong, JCAP 0408, 006 (2004) [astro-ph/0403590];

K. Enqvist, S. Hannestad and M. S. Sloth, JCAP 0502004 (2005) [astroph/0409275]; 
J. Shen, B. Wang, E. Abdalla and R. K. Su, Phys. Lett. B 609200 (2005) [hepth/0412227];

H. C. Kao, W. L. Lee and F. L. Lin, Phys. Rev. D 71123518 (2005) [astro$\mathrm{ph} / 0501487]$.

[13] Q. G. Huang and M. Li, JCAP 0408, 013 (2004) [astro-ph/0404229].

K. Enqvist and M. S. Sloth, Phys. Rev. Lett. 93, 221302 (2004) [hep-th/0406019];

K. Ke and M. Li, Phys. Lett. B 606, 173 (2005) [hep-th/0407056];

Q. G. Huang and M. Li, JCAP 0503, 001 (2005) [hep-th/0410095];

D. Pavon and W. Zimdahl, Phys. Lett. B 628, 206 (2005) [gr-qc/0505020];

B. Wang, Y. Gong and E. Abdalla, Phys. Lett. B 624, 141 (2005) [hep-th/0506069];

H. Kim, H. W. Lee and Y. S. Myung, Phys. Lett. B 632, 605 (2006) [gr-qc/0509040];

S. Nojiri and S. D. Odintsov, Gen. Rel. Grav. 38, 1285 (2006) [hep-th/0506212];

E. Elizalde, S. Nojiri, S. D. Odintsov and P. Wang, Phys. Rev. D 71, 103504 (2005) [hep-th/0502082];

B. Hu and Y. Ling, Phys. Rev. D 73, 123510 (2006) [hep-th/0601093];

H. Li, Z. K. Guo and Y. Z. Zhang, Int. J. Mod. Phys. D 15, 869 (2006) [astro$\mathrm{ph} / 0602521]$

M. R. Setare, Phys. Lett. B 642, 1 (2006) [hep-th/0609069];

M. R. Setare, Phys. Lett. B642, 421, (2006) [hep-th/0609104];

M. R. Setare, J. Zhang, X. Zhang, JCAP 0703, 007, (2007);

M. R. Setare, Eur. Phys. J. C 50, 991, (2007);

M. R. Setare, JCAP, 0701, 023 (2007);

M. R. Setare, Phys. Lett. B 648, 329, (2007).

[14] Y. Gong, Phys. Rev. D, 70, 064029, (2004).

[15] H. Kim, H. W. Lee, and Y. S. Myung, Phys. Lett. B 628, 11, (2005).

[16] D. F. Torres, Phys. Rev. D66, 043522, (2002).

[17] M. R. Setare, Phys. Lett. B 644, 99, (2007).

[18] C. Brans and C. H. Dicke, Phys. Rev. 124, 925 (1961).

[19] S. Nojiri, S. D. Odintsov, H. Stefancic, Phys. Rev. D 74, 086009, (2006); S. Nojiri, S. D. Odintsov, J. Phys. A 40, 6725, (2007); G. Cognola, E. Elizalde, S. Nojiri, S. D. Odintsov and S. Zerbini, Phys. Rev. D75 , 086002, (2007); S. Nojiri and, S. D. Odintsov, J. Phys. Conf. Ser.66, 012005, (2007).

[20] S. Capozziello, Int. J. Mod. Phys. D 11, 483 (2002); S. Capozziello, S. Carloni and A. Troisi, arXiv:astro-ph/0303041; S. M. Carroll, V. Duvvuri, M. Trodden and S. Turner, Phys. Rev. D 70 (2004) 043528.

[21] S. Nojiri and S. D. Odintsov, Phys. Rev. D 68, 123512 (2003).

[22] S. Nojiri, S. D. Odintsov and M. Sasaki, Phys. Rev. D 71, 123509 (2005); S. Nojiri, S. D. Odintsov and M. Sami, Phys. Rev. D 74, 046004, (2006); B. M. N. Carter, I. P. Neupane, Phys. Lett. B638, 94, (2006); B. M. N. Carter, and I. P. Neupane, JCAP 0606, 004, (2006); J. W. Moffat, and V. T. Toth . arXiv:0710.0364 [astro-ph]. 
[23] S. Nojiri, S. D. Odintsov, 0707.1941v2 [hep-th];S. Nojiri, S. D. Odintsov, 0710.1738v2 [hep-th]; G. Cognola, E. Elizalde, S. Nojiri, S. D. Odintsov, L. Sebastiani, S. Zerbini, 0712.4017v1 [hep-th].

[24] S. Capozziello, S. Nojiri, S. D. Odintsov and A. Troisi, Phys. Lett. B 639, 135, (2006) ; S. Nojiri and S. D. Odintsov, Phys. Rev. D74, 086005, (2006).

[25] Q. G. Huang, Y. Gong, JCAP, 0408, (2004),006.

[26] H. C. Kao, W. L. Lee and F. L. Lin, astro-ph/0501487.

[27] J. Shen, B. Wang, E. Abdalla and R. K. Su, hep-th/0412227.

[28] W. Hu and I. Sawicki, 0705.1158[astro-ph].

[29] S. Nojiri and S. D. Odintsov, Int. J. Geom. Meth. Mod. Phys. 4, 115, (2007). 